\title{
Analytical Solutions for the Elastic Circular Rod Nonlinear Wave, Boussinesq, and Dispersive Long Wave Equations
}

\author{
Shi Jing ${ }^{1}$ and Yan Xin-lii \\ ${ }^{1}$ School of Science, Chang'an University, Xi'an 710064, China \\ ${ }^{2}$ School of Science, Xian University of Architecture and Technology, Xian 710055, China \\ Correspondence should be addressed to Shi Jing; shijing-sj@163.com
}

Received 31 December 2013; Revised 23 March 2014; Accepted 25 March 2014; Published 22 April 2014

Academic Editor: Michael Meylan

Copyright (c) 2014 S. Jing and Y. Xin-li. This is an open access article distributed under the Creative Commons Attribution License, which permits unrestricted use, distribution, and reproduction in any medium, provided the original work is properly cited.

\begin{abstract}
The solving processes of the homogeneous balance method, Jacobi elliptic function expansion method, fixed point method, and modified mapping method are introduced in this paper. By using four different methods, the exact solutions of nonlinear wave equation of a finite deformation elastic circular rod, Boussinesq equations and dispersive long wave equations are studied. In the discussion, the more physical specifications of these nonlinear equations, have been identified and the results indicated that these methods (especially the fixed point method) can be used to solve other similar nonlinear wave equations.
\end{abstract}

\section{Introduction}

Nonlinear partial differential equations are widely used to describe complex phenomena in various fields of sciences such as biology, chemistry, communication, and especially many branches of physics like condensed matter physics, field theory, fluid dynamics, plasma physics, and optics, and so forth. In this paper, we consider nonlinear wave equation of a finite deformation elastic circular rod, Boussinesq equations, and dispersive long wave equations with same physical behavior. The elastic circular rod is one important component in the structures, in which the dynamics of these components are governed by double nonlinearity and double dispersion wave equation [1]. The Boussinesq equation, which was first introduced in 1871, arises in several physical applications. The dynamics of shallow water waves, which are seen in various places like sea beaches, lakes, and rivers, are governed by the Boussinesq equation. In recent years there has been much interest in some variants of the Boussinesq systems [2-6]. These coupled Boussinesq equations [7] arise in shallow water waves for two-layered fluid flow. This situation occurs when there is an accidental oil spill from a ship which results in a layer of oil floating above the layer of water. The $(2+1)$ dimensional dispersive long wave equations $[8,9]$ were first derived by Boiti as a compatibility condition for a "weak"
Lax pair. A good understanding of the solutions for these equations is very helpful to coastal and civil engineers in applying the nonlinear water model to coastal harbor design.

In the field of nonlinear science to find exact solutions for a nonlinear system is one of the most fundamental and significant studies. The evaluation of exact solutions of nonlinear wave has complicated nonlinear defects; such equations are often very difficult to be solved. Although intensive investigations have made significant progress in recent years, many methods have been proposed to construct exact solutions, such as Weierstrass elliptic function method, the homogeneous balance method, sine-cosine method, the nonlinear transformation method, the hyperbolic tangent functions finite expansion, improved mapping approach, and further extended tanh method.

The homogeneous balance method is a powerful tool to find solitary wave solutions of nonlinear partial differential equations. Fan introduced the homogeneous balance method into the search for Bäcklund transformations and obtained more solutions [10].

The mapping method is a very effective direct method to construct exact solutions of nonlinear equations. Zhang et al. make use of the auxiliary equation and the expanded mapping methods to find the new exact periodic solutions for $(2+1)$-dimensional dispersive long wave equations [11]. 
The basic idea of the fixed point methods consists in finding an iteration function, which generates successive approximations to the solution [12].

Many periodic solutions have been recently expressed in terms of various Jacobi-elliptic functions for a wide class of nonlinear evolution equations, which have been obtained by means of Jacobi elliptic expansion method [13].

The longitudinal wave equation of a finite deformation elastic circular rod, Boussinesq equations, and dispersive long wave equations are nonlinear partial differential equations of different scientific field. We find that they have the same characteristics. In this paper, the analytical solutions of the differential equations for the elastic circular rod, Boussinesq equations, and dispersive long wave equations are solved by using homogeneous balance method, Jacobi elliptic function method, fixed point method, and modified mapping method. The more physical specifications of these nonlinear equations have been identified and the results indicated.

\section{Three Types of Nonlinear Wave Equations}

The longitudinal wave equation of a finite deformation elastic circular rod is [1]

$$
u_{t t}-c_{0}^{2} u_{x x}=\left[\frac{3 E}{2 \rho} u^{2}+\frac{E}{2 \rho} u^{3}+\frac{v^{2} R^{2}}{2}\left(u_{t t}-c_{1}^{2} u_{x x}\right)\right]_{x x},
$$

where $c_{0}=\sqrt{E / \rho}$ is the longitudinal wave velocity for a linear elastic rod and $c_{1}=\sqrt{\mu / \rho}$ is the shear wave velocity. $\rho$ is the density of the material. $v$ is the Poisson ratio. $R$ is the diameter of bar. $E$ is Yong's modulus of material. $\mu$ is the elastic shear modulus of material. $t$ is the time variable.

Make the traveling wave transformation

$$
u(x, t)=u(\xi), \quad \xi=k(x-\lambda t)
$$

Substituting (2) into (1) and integrating it with respect to $\xi$ twice, we can obtain

$$
k^{2} u^{\prime \prime}+\beta_{1} u+\beta_{2} u^{2}+\beta_{3} u^{3}=N
$$

where $N$ is the second integral constant. The first integral constant is zero and

$$
\begin{gathered}
\beta_{1}=\frac{2\left(\lambda^{2}-c_{0}^{2}\right)}{v^{2} R^{2}\left(\lambda^{2}-c_{1}^{2}\right)}, \quad \beta_{2}=\frac{3 c_{0}^{2}}{v^{2} R^{2}\left(\lambda^{2}-c_{1}^{2}\right)}, \\
\beta_{3}=\frac{c_{0}^{2}}{v^{2} R^{2}\left(\lambda^{2}-c_{1}^{2}\right)} .
\end{gathered}
$$

The variant Boussinesq equations were discussed in [7]. They are coupling wave equations which can be expressed as follows:

$$
\begin{gathered}
H_{t}+(H u)_{x}+u_{x x x}=0, \\
u_{t}+H_{x}+u u_{x}=0,
\end{gathered}
$$

where $u(x, t)$ is the velocity and $H(x, t)$ is the total depth. Make the traveling wave transformation

$$
H(x, t)=H(\xi), \quad u(x, t)=u(\xi), \quad \xi=k(x-\lambda t) .
$$

Substituting (6) into (5) and integrating with respect to $\xi$, we have

$$
-\lambda H+H u+k^{2} u^{\prime \prime}=a_{1}, \quad H=\lambda u-\frac{1}{2} u^{2}+b_{1},
$$

where $a_{1}, b_{1}$ are integral constants.

Substituting the second formula of (7) into the first one and assuming $\beta_{1}=b_{1}-\lambda^{2}, \beta_{2}=(3 / 2) \lambda, \beta_{3}=-(1 / 2), N=a_{1}+$ $\lambda b_{1}$, we can obtain (3). So the solutions of (7) are equivalent to that of (3) and the second formula of (7).

$(2+1)$-dimension dispersive long wave equations were discussed in $[8,9]$. They can be written as follows:

$$
\begin{aligned}
& u_{t y}+h_{x x}+\frac{1}{2}\left(u^{2}\right)_{x y}=0, \\
& h_{t}+\left(h u+u+u_{x y}\right)_{x}=0 .
\end{aligned}
$$

Suppose that the traveling wave solutions for (8) can be given in the following forms:

$$
\begin{gathered}
u(x, y, t)=u(\xi), \quad h(x, y, t)=h(\xi), \\
\xi=x+n y+\omega t
\end{gathered}
$$

Substituting (9) into (8), we have

$$
\begin{gathered}
n u^{\prime \prime}+\left(1-n \omega^{2}\right) u-\frac{3}{2} n \omega u^{2}-\frac{n}{2} u^{3}=N_{1}, \\
h=-n \omega u-\frac{1}{2} n u^{2}
\end{gathered}
$$

where $N_{1}$ is the second integral constant.

Obviously, the first formula of (10) and (3) have the same characteristics. On the other hand, the solutions of (10) and (7) are same with each other except for the coefficients. Therefore, in following parts, the solutions of (5) are presented.

\section{Homogeneous Balance Method}

In $[14,15]$ the homogeneous balance method is used to solve nonlinear wave equations. For example, the following equation was solved in [15]:

$$
u_{t}+6 u u_{x}+u_{x x x}=0
$$

The exact solution of the following corresponding equation was given by using the same method:

$$
u_{t}-D u_{x x}+\lambda\left(u^{3}+\alpha u^{2}+\beta u\right)=0 .
$$

To solve (5), the homogeneous balance method was improved in this paper. 
Now, suppose that

$$
u(x, t)=f^{\prime} \omega_{x}+b, \quad H(x, t)=g^{\prime} \omega_{x}^{2}+c,
$$

where $f(\omega), g(\omega)$, and $\omega(x, t)$ are the undetermined functions and $b, c$ are undetermined constants.

Substituting (13) into (5), the equations can be rewritten as follows:

$$
\begin{aligned}
& \left(f^{\prime \prime} g^{\prime}+f^{\prime} g^{\prime \prime}+f^{(4)}\right) \omega_{x}^{4}+6 f^{\prime \prime \prime} \omega_{x}^{2} \omega_{2 x} \\
& \quad+f^{\prime \prime}\left(c \omega_{x}^{2}+3 \omega_{2 x}^{2}+4 \omega_{x} \omega_{3 x}\right)+g^{\prime \prime}\left(b \omega_{x}^{3}+\omega_{x}^{2} \omega_{t}\right) \\
& \quad+3 f^{\prime} g^{\prime} \omega_{x}^{2} \omega_{2 x}+f^{\prime}\left(c \omega_{2 x}+\omega_{4 x}\right) \\
& \quad+2 g^{\prime}\left(b \omega_{x} \omega_{2 x}+\omega_{x} \omega_{x t}\right)=0 \\
& \left(g^{\prime \prime}+f^{\prime} f^{\prime \prime}\right) \omega_{x}^{3}+f^{\prime \prime}\left(\omega_{x} \omega_{t}+b \omega_{x}^{2}\right)+f^{\prime 2} \omega_{x} \omega_{2 x} \\
& \quad+f^{\prime}\left(\omega_{x t}+b \omega_{2 x}\right)+2 g^{\prime} \omega_{x} \omega_{2 x}=0
\end{aligned}
$$

where $\omega_{2 x}=\omega_{x x}, \omega_{3 x}=\omega_{x x x}, \omega_{4 x}=\omega_{x x x x}$. In (14), let

$$
f^{\prime \prime} g^{\prime}+f^{\prime} g^{\prime \prime}+f^{(4)}=0, \quad g^{\prime \prime}+f^{\prime} f^{\prime \prime}=0 .
$$

Integrating the second formula of (15), we have

$$
g^{\prime}+\frac{1}{2} f^{\prime 2}=0
$$

Furthermore, we can obtain

$$
g^{\prime}=-\frac{1}{2} f^{\prime 2}, \quad g^{\prime \prime}=-f^{\prime} f^{\prime \prime} .
$$

Substituting (17) into the first formula of (15), we have

$$
f^{(4)}-\frac{3}{2} f^{\prime 2} f^{\prime \prime}=0
$$

Integrating (18), we have

$$
f^{\prime \prime \prime}-\frac{1}{2} f^{\prime 3}=0
$$

We know the solution of (19) is

$$
f=2 \ln \omega .
$$

Obviously, we have

$$
\begin{aligned}
& f^{\prime 2}=-2 f^{\prime \prime}, \quad f^{\prime} f^{\prime \prime}=-f^{\prime \prime \prime}, \\
& f^{\prime} g^{\prime}=-f^{\prime \prime \prime}, \quad f^{\prime 3}=2 f^{\prime \prime \prime} .
\end{aligned}
$$

Substituting (17) and (21) into (14), the equations can be rewritten as follows:

$$
\begin{aligned}
\omega_{x}^{2}\left(3 \omega_{2 x}+b \omega_{x}+\omega_{t}\right) f^{\prime \prime \prime} \\
+\left(c \omega_{x}^{2}+3 \omega_{2 x}^{2}+4 \omega_{x} \omega_{3 x}+2 b \omega_{x} \omega_{2 x}+2 \omega_{x} \omega_{x t}\right) f^{\prime \prime} \\
+\left(c \omega_{2 x}+\omega_{4 x}\right) f^{\prime}=0, \\
\quad \omega_{x}\left(\omega_{t}+b \omega_{x}\right) f^{\prime \prime}+\left(\omega_{x t}+b \omega_{2 x}\right) f^{\prime}=0 .
\end{aligned}
$$

Letting all coefficients of $f$ in (22) be zero, we have

$$
\begin{gathered}
3 \omega_{2 x}+b \omega_{x}+\omega_{t}=0, \\
c \omega_{x}^{2}+3 \omega_{2 x}^{2}+4 \omega_{x} \omega_{3 x}+2 b \omega_{x} \omega_{2 x}+2 \omega_{x} \omega_{x t}=0 \\
c \omega_{2 x}+\omega_{4 x}=0, \\
\omega_{t}+b \omega_{x}=0, \quad \omega_{x t}+b \omega_{2 x}=0 .
\end{gathered}
$$

From first and fifth second formula of (23), it can be obtained that

$$
\omega=\alpha(t) x+\beta(t),
$$

where $\alpha(t)$ is undetermined function and $\beta(t)$ is arbitrary function.

From second formula of (23), we have

$$
\alpha(t)=e^{-(c / 2) t+k},
$$

where $k$ is the integral constant.

Therefore, from (13), (20), (24), and (25), we know the solutions of (5) are

$$
\begin{gathered}
u_{1}(x, t)=\frac{2 \alpha(t)}{\alpha(t) x+\beta(t)}+b, \\
H_{1}(x, t)=\frac{-2 \alpha^{2}(t)}{[\alpha(t) x+\beta(t)]^{2}}+c .
\end{gathered}
$$

\section{Jacobi Elliptic Function Method}

In [16], the NLS equation and Zakharov equation were studied by using the Jacobi elliptic function method. In this paper, (1) and (5) are discussed by using this method.

\subsection{Jacobi Elliptic Sine Function Method. Let}

$$
u=a_{0}+a_{1} \operatorname{sn} \xi
$$

We know

$$
\begin{gathered}
\frac{d u}{d \xi}=a_{1} \operatorname{cn} \xi \operatorname{dn} \xi \\
\frac{d^{2} u}{d \xi^{2}}=-\left(1+m^{2}\right) a_{1} \operatorname{sn} \xi+2 m^{2} a_{1} \operatorname{sn}^{3} \xi \\
u^{2}=a_{0}^{2}+2 a_{0} a_{1} \operatorname{sn} \xi+a_{1}^{2} \operatorname{sn}^{2} \xi \\
u^{3}=a_{0}^{3}+3 a_{0}^{2} a_{1} \operatorname{sn} \xi+3 a_{0} a_{1}^{2} \operatorname{sn}^{2} \xi+a_{1}^{3} \operatorname{sn}^{3} \xi
\end{gathered}
$$

where $\operatorname{cn} \xi$ and $\operatorname{dn} \xi$ are the Jacobi elliptic cosine function and the third type Jacobi elliptic function, respectively, and $m(0<m<1)$ is module.

Substituting (27) into (3), we have

$$
\begin{aligned}
\beta_{1} a_{0} & +\beta_{2} a_{0}^{2}+\beta_{3} a_{0}^{3}-N \\
& +a_{1}\left[-k^{2}\left(1+m^{2}\right)+\beta_{1}+2 \beta_{2} a_{0}+3 \beta_{3} a_{0}^{2}\right] \operatorname{sn} \xi \\
& +a_{1}^{2}\left(\beta_{2}+3 \beta_{3} a_{0}\right) \operatorname{sn}^{2} \xi+a_{1}\left(2 k^{2} m^{2}+\beta_{3} a_{1}^{2}\right) \operatorname{sn}^{3} \xi=0 .
\end{aligned}
$$



have

Let the coefficients of all derivatives of $\operatorname{sn} \xi$ be zero and we

$$
\begin{gathered}
a_{0}=\frac{-\beta_{2}}{3 \beta_{3}}, \quad a_{1}= \pm \frac{\sqrt{2} k m}{\sqrt{-\beta_{3}}}, \\
k^{2}=\frac{1}{1+m^{2}}\left(\beta_{1}+2 \beta_{2} a_{0}+3 \beta_{3} a_{0}^{2}\right), \\
N=\beta_{1} a_{0}+\beta_{2} a_{0}^{2}+\beta_{3} a_{0}^{3} .
\end{gathered}
$$

Therefore the solution of (3) is

$$
u_{2}(x, t)=a_{0}+a_{1} \operatorname{sn} k(x-\lambda t),
$$

where $a_{0}, a_{1}$, and $k$ are denoted by (30), and $\lambda$ is arbitrary constant.

$$
\begin{aligned}
\text { When } m \rightarrow 1, \operatorname{sn} \xi & \rightarrow \tanh \xi \\
& u_{2}(x, t)=a_{0}+a_{1} \tanh k(x-\lambda t) .
\end{aligned}
$$

Thus, the solutions of (5) are (32) and the following formula:

$$
H_{2}(x, t)=b_{1}+\lambda u_{2}-\frac{1}{2} u_{2}^{2} .
$$

Assuming $\lambda=-1, b_{1}=1.5, \beta_{1}=0.5, \beta_{2}=-1.5$, and $\beta_{3}=-0.5$, it can be obtained that $a_{0}=-1, a_{1}=2$, and $k=$ 1 . The solutions of $(5)$ are $u(x, t)=-1+2 \tanh (x+t)$ and $H(x, t)=2-2 \tanh ^{2}(x+t)$. The solitary wave and behavior of the solutions $u(x, t)=-1+2 \tanh (x+t)$ and $H(x, t)=$ $2-2 \tanh ^{2}(x+t)$ are shown in Figures 1 and 2, respectively, for $0 \leq t \leq 1$ and $-10 \leq x \leq 10$. The waveform is similar to the result in [17].

\subsection{Jacobi Elliptic Cosine Function Method. Let}

$$
u=a_{0}+a_{1} \mathrm{cn} \xi
$$

Thus

$$
\begin{gathered}
u^{\prime}=-a_{1} \operatorname{sn} \xi \operatorname{dn} \xi, \quad u^{\prime \prime}=\left(2 m^{2}-1\right) a_{1} \operatorname{cn} \xi-2 m^{2} \mathrm{cn}^{3} \xi \\
u^{2}=a_{0}^{2}+2 a_{0} a_{1} \operatorname{cn} \xi+a_{1}^{2} \mathrm{cn}^{2} \xi \\
u^{3}=a_{0}^{3}+3 a_{0}^{2} a_{1} \operatorname{cn} \xi+3 a_{0} a_{1}^{2} \mathrm{cn}^{2} \xi+a_{1}^{3} \mathrm{cn}^{3} \xi .
\end{gathered}
$$

Substituting (35) into (3), we can obtain

$$
\begin{aligned}
\beta_{1} a_{0} & +\beta_{2} a_{0}^{2}+\beta_{3} a_{0}^{3}-N \\
& +a_{1}\left[k^{2}\left(2 m^{2}-1\right)+\beta_{1}+2 \beta_{2} a_{0}+3 \beta_{3} a_{0}^{2}\right] \mathrm{cn} \xi \\
& +a_{1}^{2}\left(\beta_{2}+3 \beta_{3} a_{0}\right) \mathrm{cn}^{2} \xi \\
& +a_{1}\left(-2 k^{2} m^{2}+\beta_{3} a_{1}^{2}\right) \mathrm{cn}^{3} \xi=0 .
\end{aligned}
$$

Figure 1: The solitary wave and behavior of the solutions $u(x, t)=$ $-1+2 \tanh (x+t)$ for $0 \leqslant t \leqslant 1$ and $-10 \leqslant x \leqslant 10$.

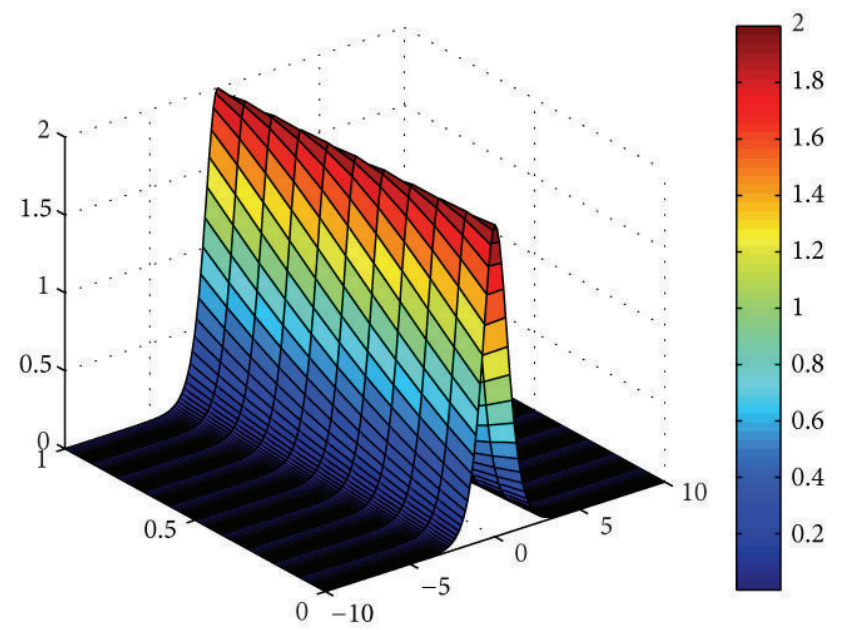

FIGURE 2: The solitary wave and behavior of the solutions $H(x, t)=$ $2-2 \tanh ^{2}(x+t)$ for $0 \leqslant t \leqslant 1$ and $-10 \leqslant x \leqslant 10$.

Let the coefficients of all derivatives of $\mathrm{cn} \xi$ be zero and we have

$$
\begin{gathered}
a_{0}=-\frac{\beta_{2}}{3 \beta_{3}}, \quad a_{1}= \pm k m \sqrt{\frac{2}{\beta_{3}}}, \\
k^{2}=\frac{\beta_{2}^{2}-3 \beta_{1} \beta_{3}}{3 \beta_{3}\left(2 m^{2}-1\right)}, \\
N=\beta_{1} a_{0}+\beta_{2} a_{0}^{2}+\beta_{3} a_{0}^{3} .
\end{gathered}
$$

Therefore, the solution of (3) is

$$
u_{3}(x, t)=a_{0}+a_{1} \operatorname{cnk}(x-\lambda t)
$$

where $a_{0}, a_{1}$ and $k$ are denoted by (37), and $\lambda$ is arbitrary constant.

The solutions of (7) are (38) and the following formula:

$$
H_{3}(x, t)=b_{1}+\lambda u_{3}(x, t)-\frac{1}{2} u_{3}^{2}(x, t) .
$$




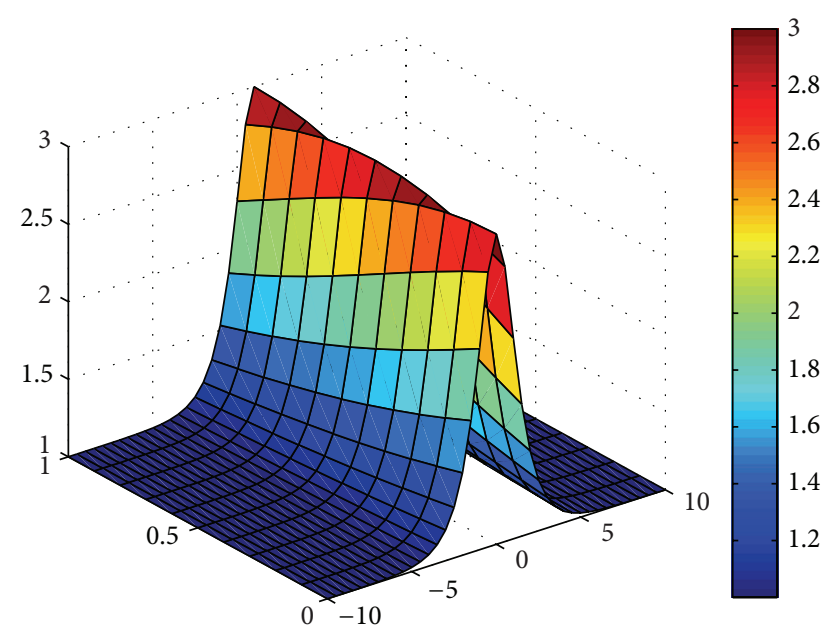

FIgURE 3: The solitary wave and behavior of the solutions $u(x, t)=$ $1+2 \operatorname{sech}(x-t)$ for $0 \leqslant t \leqslant 1$ and $-10 \leqslant x \leqslant 10$.

Taking $m \rightarrow 1, \mathrm{cn} \xi \rightarrow \operatorname{sech} \xi$, (38) can be rewritten as follows:

$$
u_{3}(x, t)=a_{0}+a_{1} \operatorname{sech} k(x-\lambda t) .
$$

Assuming $\lambda=1, \beta_{1}=0.5, \beta_{2}=-1.5$, and $\beta_{3}=0.5$, it can be obtained that $a_{0}=1, a_{1}=2$, and $k=1$. The solution of (3) is $u(x, t)=1+2 \operatorname{sech}(x-t)$. The solitary wave and behavior of the solutions are shown in Figure 3, respectively, for $0 \leq t \leq 1$ and $-10 \leq x \leq 10$.

\subsection{Third Kind of Jacobi Elliptic Function Method. Let}

$$
u=a_{0}+a_{1} \operatorname{dn} \xi
$$

Thus

$$
\begin{gathered}
u^{\prime}=-m^{2} a_{1} \operatorname{sn} \xi, \quad u^{\prime \prime}=\left(2-m^{2}\right) a_{1} \operatorname{dn} \xi-2 a_{1} \operatorname{dn}^{3} \xi \\
u^{3}=a_{0}^{3}+3 a_{0}^{2} a_{1} \operatorname{dn} \xi+3 a_{0} a_{1}^{2} \operatorname{dn}^{2} \xi+a_{1}^{3} \operatorname{dn}^{3} \xi
\end{gathered}
$$

Substituting them into (3), we can obtain

$$
\begin{aligned}
\beta_{1} a_{0} & +\beta_{2} a_{0}^{2}+\beta_{3} a_{0}^{3} \\
& -N+a_{1}\left[k^{2}\left(2-m^{2}\right)+\beta_{1}+2 \beta_{2} a_{0}+3 \beta_{3} a_{0}^{2}\right] \mathrm{dn} \xi \\
& +a_{1}^{2}\left(\beta_{2}+3 \beta_{3} a_{0}\right) \operatorname{dn}^{2} \xi+a_{1}\left(-2 k^{2}+a_{1}^{2} \beta_{3}\right) \operatorname{dn}^{3} \xi=0 .
\end{aligned}
$$

Letting the coefficients of all derivatives of $\operatorname{dn} \xi$ be zero, we have

$$
\begin{gathered}
a_{0}=\frac{-\beta_{2}}{3 \beta_{3}}, \quad a_{1}= \pm k \sqrt{\frac{2}{\beta_{3}}}, \\
k^{2}=\frac{1}{m^{2}-2}\left(\frac{3 \beta_{1} \beta_{3}-\beta_{2}^{2}}{3 \beta_{3}}\right), \\
N=\beta_{1} a_{0}+\beta_{2} a_{0}^{2}+\beta_{3} a_{0}^{3} .
\end{gathered}
$$

Therefore the solution of (3) is

$$
u_{4}(x, t)=a_{0}+a_{1} \operatorname{dn} k(x-\lambda t)
$$

where $a_{0}, a_{1}$, and $k$ are denoted by (44), and $\lambda$ is arbitrary constant.

Thus the solutions of (7) are (45) and the following formula:

$$
H_{4}(x, t)=b_{1}+\lambda u_{4}(x, t)-\frac{1}{2} u_{4}^{2}(x, t) .
$$

Letting $m \rightarrow 1$, then (45) equals (38).

\subsection{Jacobi Elliptic Function cs $\xi$ Method. Let}

$$
u=a_{0}+a_{1} \operatorname{cs} \xi, \quad \operatorname{cs} \xi \equiv \frac{\operatorname{cn} \xi}{\operatorname{sn} \xi} .
$$

Then

$$
\begin{gathered}
u^{\prime}=-a_{1}\left(1+\operatorname{cs}^{2} \xi\right) \operatorname{dn} \xi \\
u^{\prime \prime}=\left(2-m^{2}\right) a_{1} \operatorname{cs} \xi+2 a_{1} \operatorname{cs}^{3} \xi .
\end{gathered}
$$

Substituting (47) and (48) into (3), we can obtain

$$
\begin{aligned}
D= & \beta_{1} a_{0}+\beta_{2} a_{0}^{2}+\beta_{3} a_{0}^{3} \\
& -N+a_{1}\left[k^{2}\left(2-m^{2}\right)+\beta_{1}+2 \beta_{2} a_{0}+3 \beta_{3} a_{0}^{2}\right] \operatorname{cs} \xi \\
& +a_{1}^{2}\left(\beta_{2}+3 \beta_{3} a_{0}\right) \operatorname{cs}^{2} \xi+a_{1}\left(2 k^{2}+a_{1}^{2} \beta_{3}\right) \operatorname{cs}^{3} \xi .
\end{aligned}
$$

Let the coefficients in above formula be zero, and we have

$$
\begin{gathered}
a_{0}=\frac{-\beta_{2}}{3 \beta_{3}}, \quad a_{1}= \pm k^{2} \sqrt{\frac{2}{-\beta_{3}}}, \\
k^{2}=\frac{1}{m^{2}-2}\left(\frac{3 \beta_{1} \beta_{3}-\beta_{2}^{2}}{3 \beta_{3}}\right), \\
N=\beta_{1} a_{0}+\beta_{2} a_{0}^{2}+\beta_{3} a_{0}^{3} .
\end{gathered}
$$

According to (47), the solution of (3) is

$$
u_{5}(x, t)=a_{0}+a_{1} \operatorname{csk}(x-\lambda t),
$$

where $a_{0}, a_{1}$, and $k$ are denoted by (50), and $\lambda$ is arbitrary constant.

Therefore, the solutions of (7) are (51) and the following formula:

$$
H_{5}=b_{1}+\lambda u_{5}(x, t)-\frac{1}{2} u_{5}^{2}(x, t) .
$$

When $m \rightarrow 1$,

$$
u_{5}(x, t)=a_{0}+a_{1} \operatorname{csch} k(x-\lambda t) .
$$

Assuming $\beta_{1}=\left(1-n \omega^{2}\right) / n, \beta_{2}=-(3 / 2) \omega, \beta_{3}=-(1 / 2)$, $N=-\left(N_{1} / n\right)$, and $k=1$, we can obtain (3). Choosing 


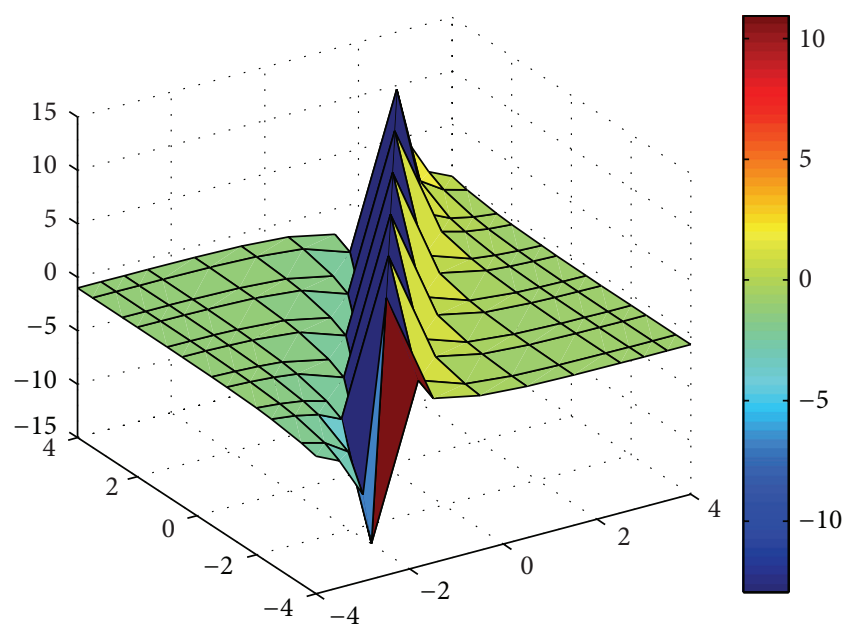

FIGURE 4: The solitary wave and behavior of the solutions $u(x, y, t)=$ $-1+2 \operatorname{csch}(x-2 / 3 y+t)$ for $t=0,-4 \leqslant x \leqslant 4$, and $-4 \leqslant y \leqslant 4$.

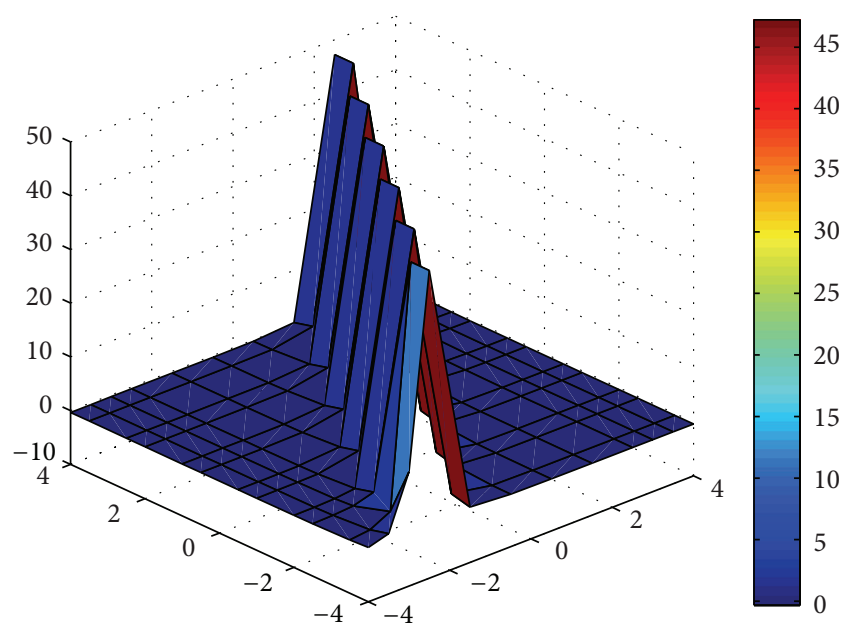

FIGURE 5: The solitary wave and behavior of the solutions $h(x, y, t)=$ $-1 / 3+4 / 3 \operatorname{csch}^{2}(x-2 / 3 y+t)$ for $t=0,-4 \leqslant x \leqslant 4$, and $-4 \leqslant y \leqslant 4$.

$n=-(2 / 3), \omega=1$, then $\beta_{1}=-(5 / 2), \beta_{2}=-(3 / 2)$. Substituting $\beta_{1}, \beta_{2}, \beta_{3}$ into (50), it can be obtained that $a_{0}=-1, a_{1}=2$, and $k=1$. The solutions of (8) are $u(x, y, t)=-1+2 \operatorname{csch}(x-(2 / 3) y+t)$ and $h(x, y, t)=$ $-(1 / 3)+(4 / 3) \operatorname{csch}^{2}(x-(2 / 3) y+t)$. The solitary wave and behavior of the solutions $u(x, t)=-1+2 \operatorname{csch}(x-(2 / 3) y+t)$ and $h(x, y, t)=-(1 / 3)+(4 / 3) \operatorname{csch}^{2}(x-(2 / 3) y+t)$ are shown in Figures 4 and 5, respectively, for $t=0,-4 \leq x \leq 4$, $-4 \leq y \leq 4$, and $x-(2 / 3) y \neq 0$.

\section{Fixed Point Method}

In general, fixed point theory can be divided into two types. The first type is only used to discuss the existence of solution. The second type is used not only to discuss the existence and uniqueness of solution but also to search the fixed point. We are more interested in the second type.
Lemma 1 (see [18-20]). Suppose $E$ is a real Banach space which has normal cone. Consider $u_{0}, v_{0} \in E, u_{0}<v_{0}$. The operator $A:\left[u_{0}, v_{0}\right] \times\left[u_{0}, v_{0}\right] \rightarrow E$ is mixed monotone and satisfies

(1)

$$
u_{0} \leq A\left(u_{0}, v_{0}\right), \quad A\left(v_{0}, u_{0}\right) \leq v_{0}
$$

(2) for all $u, v\left(u_{0} \leq u \leq v \leq v_{0}\right), \exists$ constant $\alpha \in(0,1)$, satisfies

$$
\|A(v, u)-A(u, v)\| \leq \alpha\|v-u\| .
$$

Then there exists a unique $\bar{u}\left(\bar{u} \in\left[u_{0}, v_{0}\right]\right)$ which satisfies

$$
A(\bar{u}, \bar{u})=\bar{u}
$$

where $\bar{u}$ is called the fixed point of $A$.

In $\left[u_{0}, v_{0}\right]$ for all $w_{0}$, letting $w_{n}=A\left(w_{n-1}, w_{n-1}\right), n=$ $1,2, \ldots$, we have

$$
\bar{u}=\lim _{n \rightarrow \infty} w_{n}
$$

In this paper, $E$ is taken as a continuous function space $C[0,1]$ which is defined in the closed interval $[0,1]$. Introducing equivalent norm, it is easy to know that $C[0,1]$ is real Banach space which has normal cone. Let

$$
u_{0}=0, \quad v_{0}=1
$$

Integrating (3) with respect to $\xi$, we can obtain

$$
\frac{d u}{d \xi}=-k^{-2} \int_{0}^{\xi}\left[\beta_{1} u(s)+\beta_{2} u^{2}(s)+\beta_{3} u^{3}(s)\right] d \xi+N \xi+C_{1}
$$

where $C_{1}$ is integral constant.

Integrating the above formula with respect to $\xi$ and using the formula in [12], we have

$$
\begin{aligned}
u= & -k^{-2} \int_{0}^{\xi}(\xi-s)\left[\beta_{1} u(s)+\beta_{2} u^{2}(s)+\beta_{3} u^{3}(s)\right] d s \\
& +\frac{1}{2} N \xi^{2}+C_{1} \xi+C_{2},
\end{aligned}
$$

where $C_{2}$ is integral constant.

If $\beta_{2}>0, \beta_{3}>0$ (i.e., $\lambda^{2}>c_{1}$ ), and $\beta_{1}<0$ (i.e., $\lambda^{2}<c_{0}^{2}$ ), suppose

$$
\begin{aligned}
A(u, v)= & -k^{-2} \int_{0}^{\xi}(\xi-s)\left[\beta_{1} u(s)+\beta_{2} v^{2}(s)+\beta_{3} v^{3}(s)\right] d s \\
& +\frac{1}{2} N \xi^{2}+C_{1} \xi+C_{2} .
\end{aligned}
$$

Theorem 2. In (61), supposing $\beta_{2}>0, \beta_{3}>0, \beta_{1}<0, C_{1} \geq 0$, $C_{2} \geq 0$, and $k^{-2} \leq N /\left(\beta_{2}+\beta_{3}\right) \leq 2\left(1+C_{1}+C_{2}\right) /\left(\beta_{2}+\beta_{3}-\beta_{1}\right)$, there exists unique fixed point $\bar{u} \in\left[u_{0}, v_{0}\right]$ for the operator $A$ which is defined by (61). Then (57) is true. 
Proof. Obviously, $A:\left[u_{0}, v_{0}\right] \times\left[u_{0}, v_{0}\right] \rightarrow C[0,1]$ is mixed monotone. Consider

$$
\begin{aligned}
A\left(u_{0}, v_{0}\right) & =A(0,1) \\
& =\frac{1}{2}\left[N-k^{-2}\left(\beta_{2}+\beta_{3}\right)\right] \xi^{2}+C_{1} \xi+C_{2} \geq 0=u_{0} \\
A\left(v_{0}, u_{0}\right) & =\frac{1}{2}\left(N-\beta_{1} k^{-2}\right) \xi^{2}+C_{1} \xi+C_{2} \\
& \leq \frac{1}{2} N\left(1-\frac{\beta_{1}}{\beta_{2}+\beta_{3}}\right)+C_{1}+C_{2} \leq 1=v_{0} .
\end{aligned}
$$

Thus $A$ satisfies the term (1) of Lemma 1.

For all $u, v \in\left[u_{0}, v_{0}\right], u \leq v$,

$$
\begin{aligned}
& A(v, u)-A(u, v) \\
& =-k^{-2} \int_{0}^{\xi}(\xi-s) \\
& \times\left[\beta_{1} v(s)+\beta_{2} u^{2}(s)+\beta_{2} u^{3}(s)\right. \\
& \left.-\beta_{1} u(s)-\beta_{2} v^{2}(s)-\beta_{3} v^{3}(s)\right] d s \\
& =k^{-2} \int_{0}^{\xi}(\xi-s) e^{M s} e^{-M s}[v(s)-u(s)] \\
& \times\left\{-\beta_{1}+\beta_{2}[v(s)+u(s)]\right. \\
& \left.+\beta_{3}\left[v^{2}(s)+u(s) v(s)+u^{2}(s)\right]\right\} d s \\
& \leq\left(-\beta_{1}+2 \beta_{2}+3 \beta_{3}\right) k^{-2}\|v-u\| \int_{0}^{\xi} e^{M S} d s \\
& \leq \frac{-\beta_{1}+2 \beta_{2}+3 \beta_{3}}{k^{2} M}\|v-u\| e^{M \xi} .
\end{aligned}
$$

Then, we have

$$
\|A(v, u)-A(u, v)\| \leq \alpha,
$$

where $\alpha=\left(-\beta_{1}+2 \beta_{2}+3 \beta_{3}\right) /\left(k^{2} M\right)$, and when $M$ is large enough, $\alpha \in(0,1)$.

Therefore, operator $A$ which is defined by (61) satisfies the term (2) of Lemma 1. The proof is completed.

Obviously, the fixed point of the operator defined by (61) is equivalent to the solution of (3). We denote

$$
u_{6}(x, t)=\bar{u}(\xi)
$$

By using the classical iteration algorithms, such as Mann iteration method, Ishikawa iteration method, and Noor iteration method, the solutions can be obtained. Then, the traveling wave solutions of (5) are (65) and the following formula:

$$
H_{6}(x, t)=b_{1}+\lambda u_{6}(x, t)-\frac{1}{2} u_{6}^{2}(x, t)
$$

\section{Modified Mapping Method}

The modified mapping method was introduced and the nonlinear evolution equation was solved in [21, 22]. In [22], several hundreds of Jacobi elliptic function expansion solutions were obtained. We also use this method in this paper.

Let

$$
u=A_{0}+A_{1} f+B_{1} f^{-1},
$$

where $f^{\prime \prime}=p f+q f^{3} \Leftrightarrow f^{\prime 2}=r+p f^{2}+(1 / 2) q f^{4} . r, p$, and $q$ are constants.

Thus

$$
\begin{gathered}
u^{\prime}=A_{1} f^{\prime}-B_{1} f^{-2} f^{\prime} \\
u^{\prime \prime}=A_{1} p f+A_{1} q f^{3}+B_{1} p f^{-1}+2 B_{1} r f^{-3}, \\
u^{2}=A_{0}^{2}+2 A_{1} B_{1}+2 A_{0} A_{1} f+2 A_{0} B_{1} f^{-1}+A_{1}^{2} f^{2}+B_{1}^{2} f^{-2}, \\
u^{3}=A_{0}^{3}+6 A_{0} A_{1} B_{1}+3 A_{1}\left(A_{0}^{2}+A_{1} B_{1}\right) f \\
+3 A_{0} A_{1}^{2} f^{2}+A_{1}^{3} f^{3}+3 B_{1}\left(A_{0}^{2}+A_{1} B_{1}\right) f^{-1} \\
+3 A_{0} B_{1}^{2} f^{-2}+B_{1}^{3} f^{-3} .
\end{gathered}
$$

Substituting (68) into (3), we have

$$
\begin{aligned}
\beta_{1} A_{0} & +\beta_{2}\left(A_{0}^{2}+2 A_{1} B_{1}\right)+\beta_{3}\left(A_{0}^{3}+6 A_{0} A_{1} B_{1}\right)-N \\
& +A_{1}\left[k^{2} p+\beta_{1}+\beta_{2} A_{0}+3 \beta_{3}\left(A_{0}^{2}+A_{1} B_{1}\right)\right] f \\
& +A_{1}^{2}\left(\beta_{2}+3 \beta_{3} A_{0}\right) f^{2}+A_{1}\left(k^{2} q+\beta_{3} A_{1}^{2}\right) f^{3} \\
& +B_{1}\left[k^{2} p+\beta_{1}+2 \beta_{2} A_{0}+3 \beta_{3}\left(A_{0}^{2}+A_{1} B_{1}\right)\right] f^{-1} \\
& +B_{1}^{2}\left(\beta_{2}+3 \beta_{3} A_{0}\right) f^{-2}+B_{1}\left(2 k^{2} r+\beta_{3} B_{1}^{2}\right) f^{-3}=0 .
\end{aligned}
$$

Notice that we get the same algebraic equations when the coefficients of $f, f^{-1}$ and $f^{2}, f^{-2}$ in (69) are zero. Therefore there are only five algebra equations. Solve them and we have

$$
\begin{gathered}
A_{0}=\frac{-\beta_{2}}{3 \beta_{3}}, \quad A_{1}= \pm k \sqrt{\frac{-q}{\beta_{3}}}, \quad B_{1}= \pm k \sqrt{\frac{-2 r}{\beta_{3}}}, \\
k= \pm \sqrt{\frac{\beta_{2}^{2}-3 \beta_{1} \beta_{3}-3 k^{2} \beta_{3} \sqrt{2 q r}}{3 \beta_{3} p}} .
\end{gathered}
$$

In order to make $A_{1}, B_{1}$ be real number, $q$ and $r$ should be different signs with $\beta_{3}$.

(i) Choosing $r=1, p=-2$, and $q=2$, we have $f=\tanh \xi$. Then the solitary wave solutions of (3) are

$$
u_{7}(x, t)=A_{0}+A_{1} \tanh \xi+B_{1} \tanh ^{-1} \xi .
$$




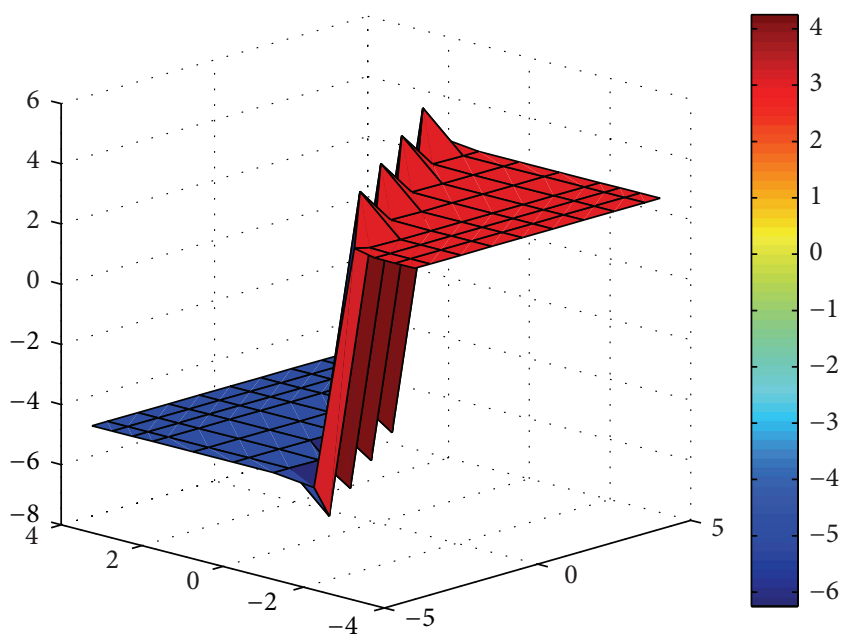

FIGURE 6: The solitary wave and behavior of the solutions $u(x, y, t)=$ $-1+2 \tanh (x-2 y+t)+2 \tanh ^{-1}(x-2 y+t)$ for $t=0,-4 \leqslant x \leqslant 4$, and $-4 \leqslant y \leqslant 4$.

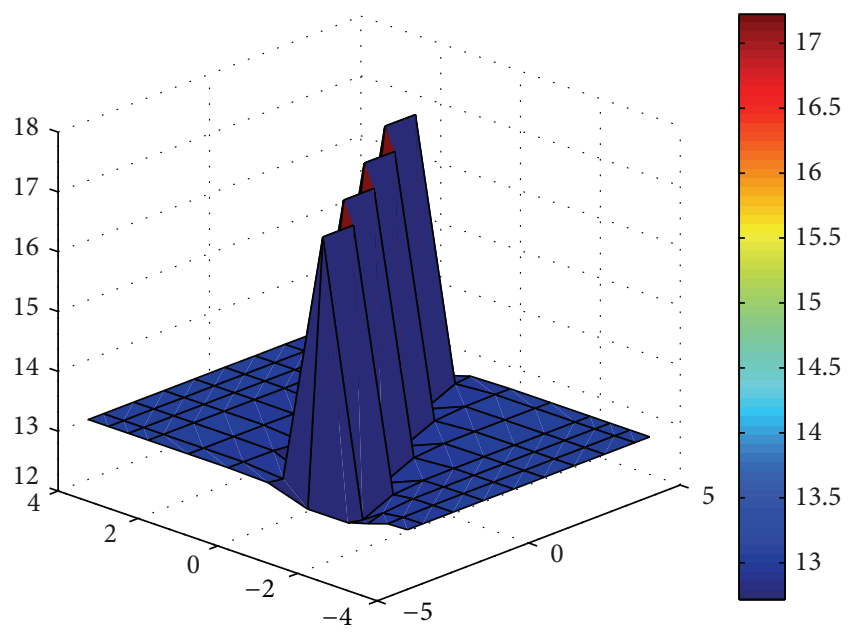

FIGURE 7: The solitary wave and behavior of the solutions $h(x, y, t)=$ $7+4 \tanh ^{2}(x-2 y+t)+4 \tanh ^{-2}(x-2 y+t)$ for $t=0,-4 \leqslant x \leqslant 4$, and $-4 \leqslant y \leqslant 4$.

Assuming $n=-2, \omega=1, k=1$, and $\beta_{3}=-(1 / 2)$, it can be obtained that $\beta_{1}=-(3 / 2), \beta_{2}=-(3 / 2), A_{0}=-1$, $A_{1}=2$, and $B_{1}=2$. The solutions of $(8)$ are $u(x, y, t)=-1+$ $2 \tanh (x-2 y+t)+2 \tanh ^{-1}(x-2 y+t)$ and $h(x, y, t)=7+$ $4 \tanh ^{2}(x-2 y+t)+4 \tanh ^{-2}(x-2 y+t)$. The solitary wave and behavior of them are shown in Figures 6 and 7, respectively, for $t=0,-4 \leq x \leq 4,-4 \leq y \leq 4$, and $x-2 y \neq 0$.

(ii) Choosing $r=1, p=-\left(1+m^{2}\right)$, and $q=2 m^{2}$, thus

$$
f=\operatorname{sn} \xi
$$

where $m(0<m<1)$ is the module of Jacobi elliptic function.

Therefore the expansion solutions of (3) are

$$
u_{8}(x, t)=A_{0}+A_{1} \operatorname{sn} \xi+B_{1} \operatorname{sn}^{-1} \xi .
$$

Letting $m \rightarrow 1$, then (73) equals (71).

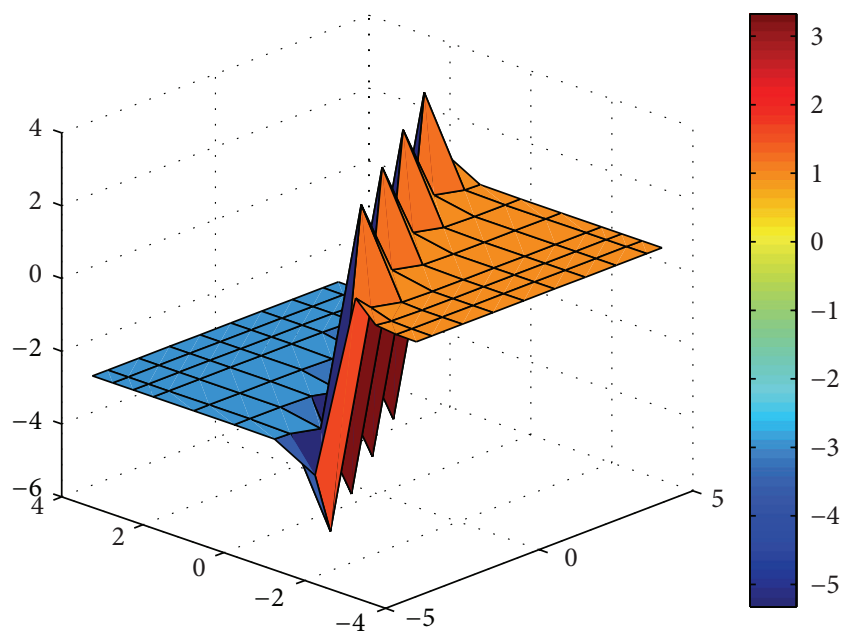

FIGURE 8: The solitary wave and behavior of the solutions $u(x, y, t)=$ $-1+\tanh (x-2 y+t) /(1+\operatorname{sech}(x-2 y+t))+(1+\operatorname{sech}(x-2 y+$ $t)) / \tanh (x-2 y+t)$ for $t=0,-4 \leqslant x \leqslant 4$, and $-4 \leqslant y \leqslant 4$. have

(iii) Choosing $r=1 / 4, p=\left(m^{2}-2\right) / 2$, and $q=m^{2} / 2$, we

$$
f=\frac{\operatorname{sn} \xi}{(1+\operatorname{dn} \xi)}
$$

Therefore the solutions of (3) are

$$
u_{9}(x, t)=A_{0}+\frac{A_{1} \operatorname{sn} \xi}{1+\operatorname{dn} \xi}+\frac{B_{1}(1+\operatorname{dn} \xi)}{\operatorname{sn} \xi},
$$

where $A_{0}, A_{1}, B_{1}$ of $u_{i}(i=7,8,9)$ are denoted by $(70)$.

Taking $m \rightarrow 1$, (75) can be rewritten as follows:

$$
u_{9}(x, t)=A_{0}+\frac{A_{1} \tanh \xi}{1+\operatorname{sech} \xi}+\frac{B_{1}(1+\operatorname{sech} \xi)}{\tanh \xi} .
$$

Assuming $n=-2, \omega=1, k=1$, and $\beta_{3}=-(1 / 2)$, it can be obtained that $\beta_{1}=-(3 / 2), \beta_{2}=-(3 / 2), A_{0}=$ $-1, A_{1}=1$, and $B_{1}=1$. The solutions of $(8)$ are $u(x$, $y, t)=-1+\tanh (x-2 y+t) /(1+\operatorname{sech}(x-2 y+t))+(1+$ $\operatorname{sech}(x-2 y+t)) / \tanh (x-2 y+t)$ and $h(x, y, t)=1+$ $[\tanh (x-2 y+t) /(1+\operatorname{sech}(x-2 y+t))]^{2}+[(1+\operatorname{sech}(x-$ $2 y+t)) / \tanh (x-2 y+t)]^{2}$. The solitary wave and behavior of them are shown in Figures 8 and 9, respectively, for $t=0$, $-4 \leq x \leq 4,-4 \leq y \leq 4$, and $x-2 y \neq 0$.

\section{Summary and Conclusions}

In summary, by using different methods, nonlinear wave equation of a finite deformation elastic circular rod, Boussinesq equations, and dispersive long wave equations are solved in this paper, and several analytical solutions are obtained. Kink soliton solutions are obtained when we use the homogenous balance method. The Jacobi elliptic function method is utilized and periodic solutions are obtained. When $m \rightarrow 1$, the solutions reduce to solitary solution. Their wave forms are the bell type, kink type, exotic type, and 


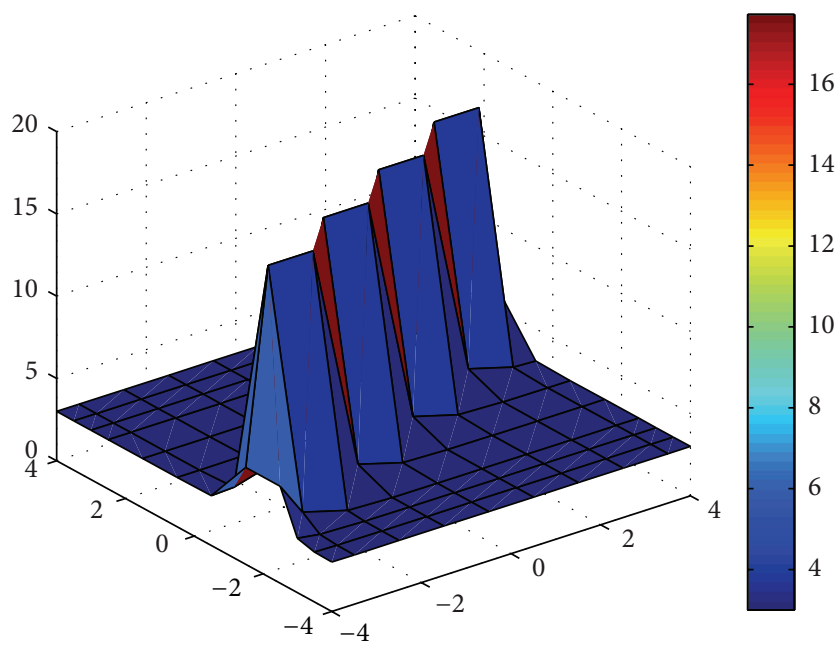

Figure 9: The solitary wave and behavior of the solutions $h(x, y, t)=1+\tanh ^{2}(x-2 y+t) /(1+\operatorname{sech}(x-2 y+t))^{2}+$ $(1+\operatorname{sech}(x-2 y+t))^{2} / \tanh ^{2}(x-2 y+t)$ for $t=0,-4 \leqslant x \leqslant 4$, and $-4 \leqslant y \leqslant 4$.

peakon type. By using the modified mapping method, peakon solutions of (3), (5), (8) are obtained. On the other hand, after proving the existence and uniqueness of fixed point of operator $A$, we can use the classical iteration algorithms to get the solutions. The method has wide application and can be used to solve other nonlinear wave equations. Fixed point method may be significant and important for the explanation of some special physical problems whose analytical solution cannot be obtained. The results indicate the following. First, the natural phenomena and the physical properties of the equations are abundant, which should be further discovered. Second, there are many kinds of methods for solving these equations. Third, the arbitrary constant can be determined by the initial and boundary conditions and we can obtain the exact solutions for certain engineering or scientific problems. It is shown that the methods, proposed in this paper for three types of nonlinear wave equations, are feasible for determining exact solutions of other nonlinear equations.

\section{Conflict of Interests}

The authors declare that there is no conflict of interests regarding the publication of this paper.

\section{Acknowledgment}

This work was supported by the Special Fund for Basic Scientific Research of Central Colleges (Grant no. CHD2009JC140).

\section{References}

[1] Z. F. Liu and S. Y. Zhang, "Solitary waves in finite deformation elastic circular rod," Journal of Applied Mathematics and Mechanics, vol. 27, no. 10, pp. 1255-1260, 2006.
[2] E. V. Krishnan, S. Kumar, and A. Biswas, "Solitons and other nonlinear waves of the Boussinesq equation," Nonlinear Dynamics. An International Journal of Nonlinear Dynamics and Chaos in Engineering Systems, vol. 70, no. 2, pp. 1213-1221, 2012.

[3] A. Biswas, M. Song, H. Triki et al., "Solitons, Shockwaves, conservation laws and bifurcation analysis of boussinesq equation with power law nonlinearity and dual-dispersion," Journal of Applied Mathematics and Information Science, vol. 8, no. 3, pp. 949-957, 2014.

[4] A. Ja'afar, M. Jawad, M. D. Petkovic, and A. Biswas, "Soliton solutions to a few coupled nonlinear wave equations by tanh method," The Iranian Journal of Science and Technology A, vol. 37, no. 2, pp. 109-115, 2013.

[5] J. F. Zhang, "Multi-solitary wave solutions for variant Boussinesq equations and Kupershmidt equations," Journal of Applied Mathematics and Mechanics, vol. 21, no. 2, pp. 171-175, 2000.

[6] M. Zhang, Q. Liu, J. Wang, and K. Wu, "A new supersymmetric classical Boussinesq equation," Chinese Physics B, vol. 17, no. 1, pp. 10-16, 2008.

[7] Y. B. Yuan, D. M. Pu, and S. M. Li, "Bifurcations of travelling wave solutions in variant Boussinesq equations," Journal of Applied Mathematics and Mechanics, vol. 27, no. 6, pp. 716-726, 2006.

[8] Sirendaoreji and S. Jiong, "Auxiliary equation method for solving nonlinear partial differential equations," Physics Letters A, vol. 309, no. 5-6, pp. 387-396, 2003.

[9] Taogetusang and Sirendaoreji, "New types of exact solitary wave solutions for $(2+1)$-dimensional dispersive long-wave equations and combined KdV-Burgers equation," Chinese Journal of Engineering Mathematics, vol. 23, no. 5, pp. 943-946, 2006.

[10] E. G. Fan and H. Q. Zhang, "Solitary wave solutions of a class of nonlinear wave equations," Acta Physica Sinica, vol. 46, no. 7, pp. 1254-1258, 1997.

[11] W. L. Zhang, G. J. Wu, M. Zhang, J. M. Wang, and J. H. Han, "New exact periodic solutions to $(2+1)$-dimensional dispersive long wave equations," Chinese Physics B, vol. 17, no. 4, pp. 1156$1164,2008$.

[12] Z. Bartoszewski, "Solving boundary value problems for delay differential equations by a fixed-point method," Journal of Computational and Applied Mathematics, vol. 236, no. 6, pp. 1576-1590, 2011.

[13] Z. T. Fu, S. K. Liu, S. D. Liu, and Q. Zhao, "New Jacobi elliptic function expansion and new periodic solutions of nonlinear wave equations," Physics Letters A, vol. 290, no. 1-2, pp. 72-76, 2001.

[14] M. L. Wang, Y. B. Zhou, and Z. B. Li, "Application of a homogeneous balance method to exact solutions of nonlinear equations in mathematical physics," Physics Letters A: General, Atomic and Solid State Physics, vol. 216, no. 1-5, pp. 67-75, 1996.

[15] E. G. Fan and H. Q. Zhang, "The homogeneous balance method for solving nonlinear soliton equations," Acta Physica Sinica, vol. 47, no. 3, pp. 353-362, 1998.

[16] S. D. Liu, Z. T. Fu, S. K. Liu, and Q. Zhao, "Enveloping periodic solutions to nonlinear wave equations with Jacobi elliptic functions," Acta Physica Sinica, vol. 51, no. 4, pp. 718$722,2002$.

[17] A. J. M. Jawad, M. D. Petković, P. Laketa, and A. Biswas, "Dynamics of shallow water waves with Boussinesq equation," Scientia Iranica B, vol. 20, no. 1, pp. 179-184, 2013.

[18] X. L. Yan, "Existence and uniqueness theorems of solution for symmetric contracted operator equations and their applications," Chinese Science Bulletin, vol. 36, no. 10, pp. 800-805, 1991. 
[19] J. P. Hao and X. L. Yan, "Exact solution of large deformation basic equations of circular membrane under central force," Journal of Applied Mathematics and Mechanics, vol. 27, no. 10, pp. 1169-1172, 2006.

[20] X. L. Yan, Exact Solution of Nonlinear Equations, Economic Scientic Press, Beijing, China, 2004.

[21] Y.-Z. Peng, "Exact solutions for some nonlinear partial differential equations," Physics Letters A, vol. 314, no. 5-6, pp. 401-408, 2003.

[22] L. X. Gong, "Some new exact solutions via the Jacobi elliptic functions to the nonlinear Schrödinger equation," Acta Physica Sinica, vol. 55, no. 9, pp. 4414-4419, 2006. 


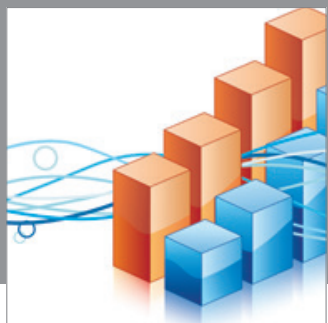

Advances in

Operations Research

mansans

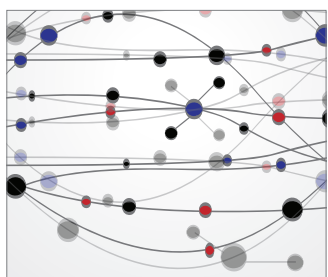

The Scientific World Journal
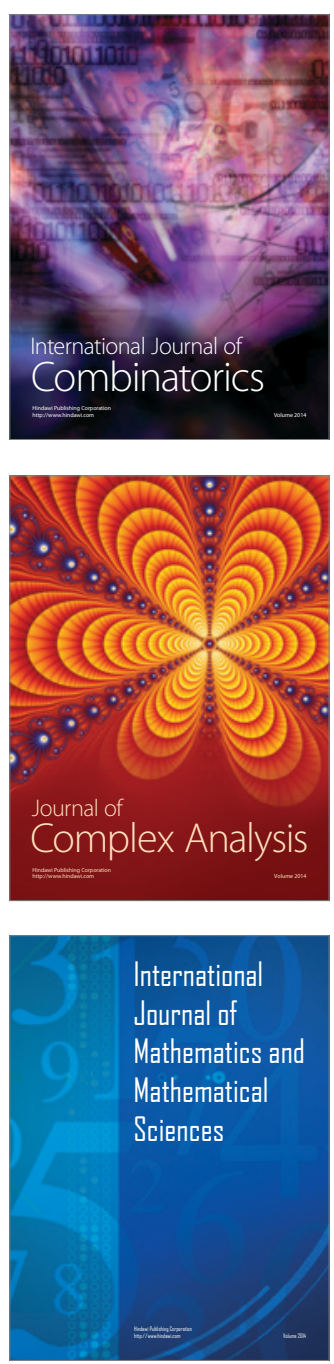
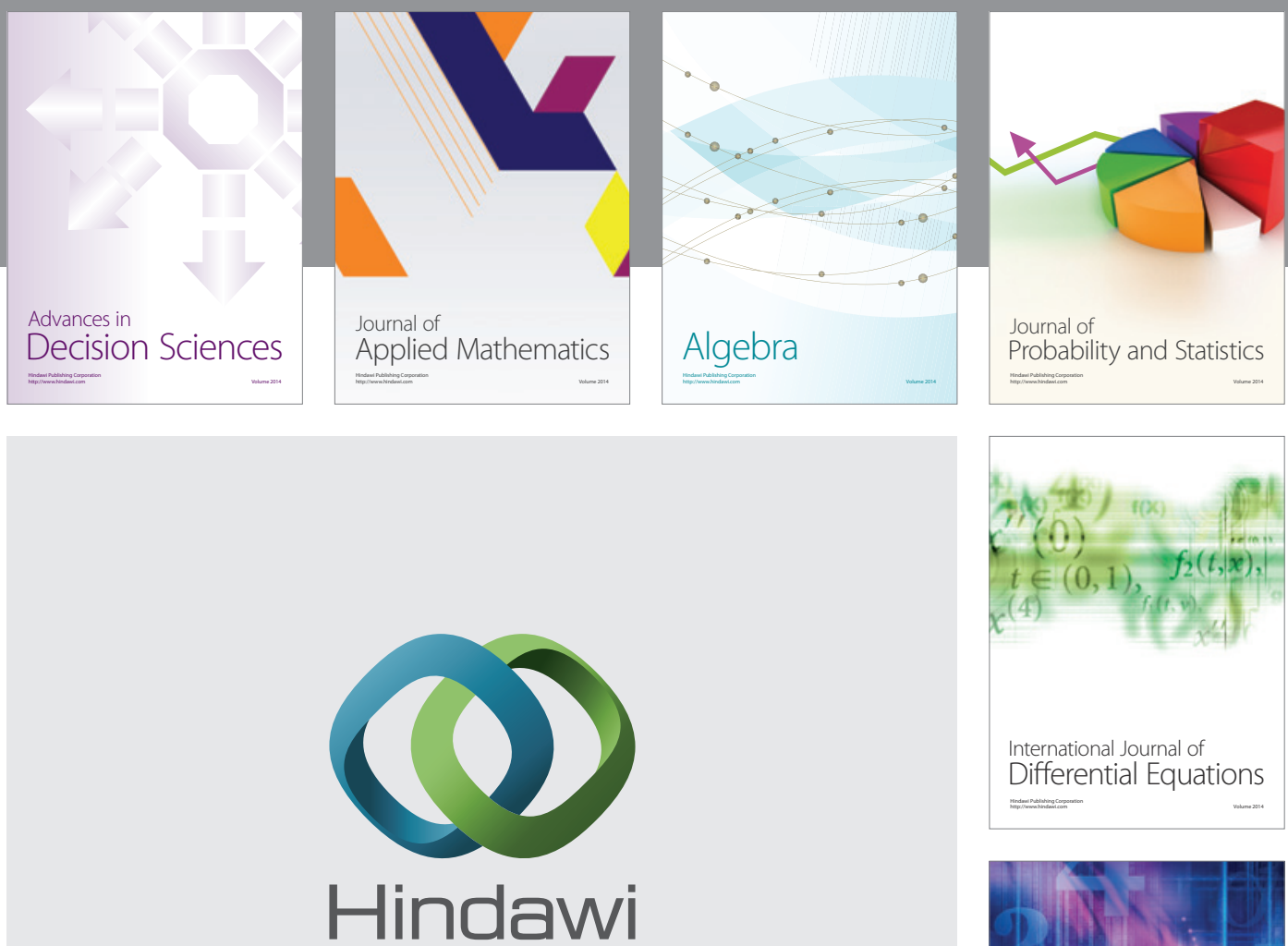

Submit your manuscripts at http://www.hindawi.com
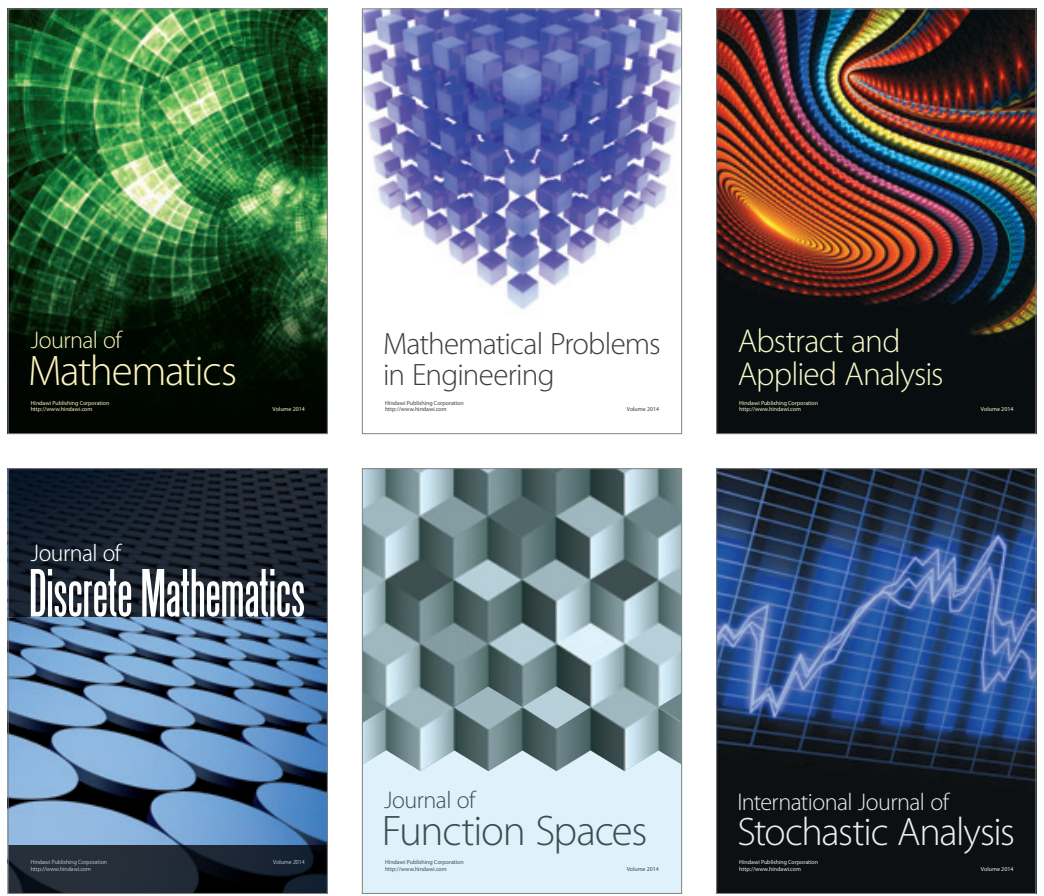

Journal of

Function Spaces

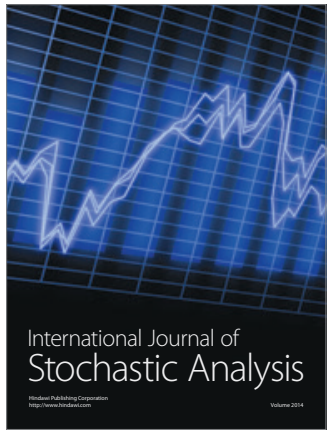

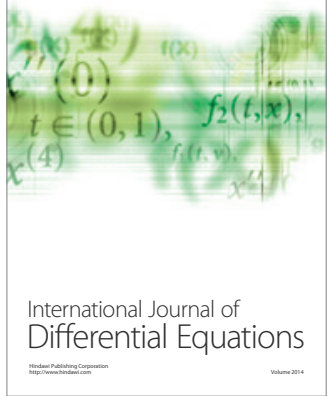
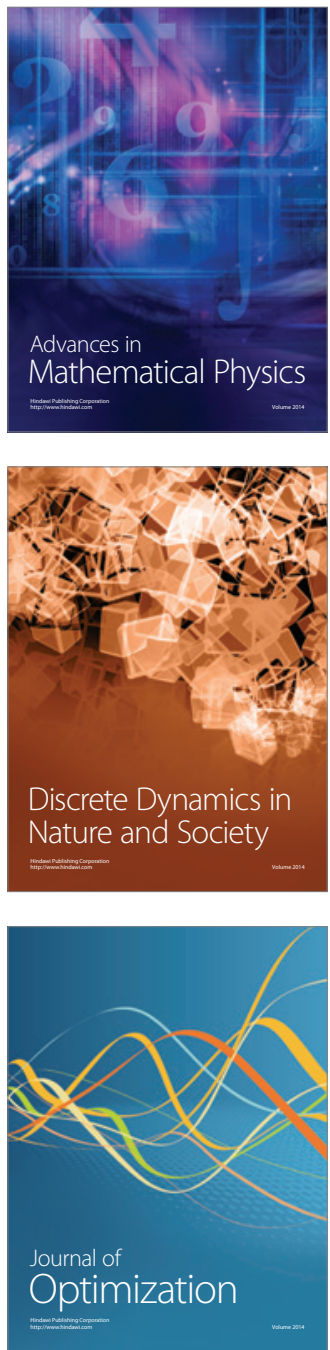